\title{
CONTROLLED X-SEGREGATION AT MEIOSIS IN TEGENARIA
}

\author{
S. H. REVELL \\ John Innes Horticulturol Institution, Merton
}

Received 10.vi.47

\section{INTRODUCTION}

OBSERvatrons made upon a system operating under abnormal conditions are often of value in finding out how its normal function is controlled. This method of attack has been most profitable in cytology. In the present paper an abnormal type of meiosis, apparently characteristic of male spiders, is described, and its unusual features discussed with particular regard to the general problems of chromosome polarisation and chromosome movement on the spindle.

The Aranee have received little attention from cytologists. Wallace (I909) first reported the multiple sex chromosome mechanism of an XXO type in Agelena nevia males, and this was confirmed by Painter (19I4) in twelve widely representative genera. Recently Hard (I939) described the same mechanism in Schizocosa crassipes. Both X's are said to be markedly heterochromatic and they do not form chiasmata, though they remain side by side throughout the first meiotic division. At first anaphase they move together to the same pole. It is the adaptation of meiosis to this unusual sex chromosome mechanism that makes the material of special interest.

\section{MATERIALS AND METHODS}

Three common British household spiders were examined-Tegenaria atrica, $T$. derhamii and $T$. domestica. All material was taken from the wild.

Two techniques were adopted. Squashes in Lacmoid stain (Darlington and La Cour, 1942), made after fixation of whole testes in acetic alcohol, were found to be excellent for the meiotic and spermatogonial chromosomes in all three species. Some mitoses were also obtained from ovary tissue in $T$. domestica by this technique. To follow the orientation of the prophase and metaphase chromosomes to the centrosomes at meiosis some material was fixed in $2 \mathrm{BD}$, sectioned at $20 \mu$ and stained in Crystal Violet. 


\section{MITOTIC CHROMOSOMES}

In all three species of Tegenaria examined the autosome number is forty, but while $T$. atrica males have the characteristic XXO complement that has previously been reported in the Aranee, the species $T$. derhamii and $T$. domestica have three-X males. Several somatic counts of forty autosomes and the expected number of six $\mathrm{X}$ 's were obtained from $T$. domestica females.

In spermatogonial metaphases the pairs of autosomes and the two or three X's show strong somatic pairing. The autosomes in each species are indistinguishable; they vary little in length and all have centromeres apparently terminal. The X's can be recognised by their greater length ; and at meiosis, when they are together, differences in their length can also be detected (pl. I, figs. 4, 5, 6 and 7).

\section{MEIOSIS \\ (i) General}

The course of meiosis in the three species is essentially the same and they are therefore described together. The investigation was, however, based primarily upon observations on the two-X species, T. atrica, because it was found to be the most favourable material.

During the pre-meiotic resting stage the autosome number of forty pro-chromosomes can be seen, diffusely outlined and evenly distributed round the nuclear membrane. The X's are already paired and are strongly heterochromatic, being super-condensed and deeply stained. Their outline is clearly defined, in marked contrast to the prochromosomes which, if they represent heterochromatic autosome segments, are evidently not so heavily charged with nucleic acid as the X's.

At leptotene each autosome thread has a heterochromatic chromomere at one end, which lies against the nuclear membrane. These chromomeres are evenly spaced at first ; but subsequently they move round to concentrate on one side of the nucleus, presumably determined by the centrosomes, which are, however, not resolvable at this stage. The leptotene threads are otherwise weakly stained and have been unorientated hitherto. The effect of this migration of their terminal chromomeres is to polarise each thread so that it lies across the nucleus and parallel to its fellows.

When polarisation is completed autosome pairing begins, which is rapid and always complete. By the time of pachytene the terminal heterochromatin has lost its differential charge. At diplotene one chiasma is formed in each bivalent : no variation in chiasma number and no failure of chiasma formation was seen. There is a wide range in the point of origin of the chiasmata in each early diplotene nucleus, but the morphological similarity of the bivalents makes it impossible to be certain that this represents a random formation (pl. I, figs. 2-6). 
There appears to be no terminal movement of the chiasmata during prophase.

The sex chromosomes do not form chiasmata. They remain side by side during the first meiotic division, and though they are already split at pachytene (pl. I, fig. I) they are all at the same pole at first anaphase and do not divide. During interphase their pairing lapses (pl. I, fig. 8), though they remain condensed, and at the second division they come on to the plate separately and divide mitotically. The $\mathrm{X}$ 's are visible in the resting nuclei of half the newly formed spermatids. During the first division in the three- $\mathrm{X}$ species each $\mathrm{X}$ is always straight and in such a position that it is paired to both the others. In $T$. atrica, however, the X's are sometimes relationally coiled at pachytene.

In all three species the X's show properties characteristic of heterochromatin. They are often sticky, and the relational coiling that they show in T. atrica may be due to their sticking together. By the time of spindle formation they are undercharged, and it is in this condition that differences in their length can be most easily seen. Reports on other genera have stated that the X's are of exactly equal size, but this is not the case in Tegenaria. In lacmoid squashes of $\mathcal{T}$. atrica cells at metaphase they are 7 and $8 \mu$ long, and in $\mathcal{T}$. derhamii and $T$. domestica between 5 and $6 \mu$. At interphase and in the spermatids they are super-condensed.

\section{(ii) Polarisation in the nucleus}

Polarisation plays an important part in meiosis in Tegenaria. I shall now relate the data already given to the changes that occur in chromosome orientation, summarised in the text fig.

The centrosomes cannot be seen at the time of the concentration of chromosome ends prior to zygotene. At pachytene they are clearly visible, lying in a line at right angles to the nucleus in what we may call proximal and distal positions (pl. II, fig. 9). Each chromosome association is polarised with one of its ends towards them. The attraction is preferential, and always gives the same polarisation pattern, in which the X's are in a central position nearest the centrosomes (pl. I, fig. I). The only variation observed was the occasional failure of one or two of the autosome bivalents to polarise.

Polarisation does not lapse at diplotene as is usual in animal meiosis but, instead, continues throughout the rest of prophase. As the bivalents begin to condense a space is left on the side of the nucleus opposite to the centrosomes, and here the occasional unpolarised bivalents can be seen, pushed as it were against the nuclear membrane.

At about the end of diplotene the centrosomes begin to move apart ; each continues to act as a polarisation centre and the chromosomes therefore separate into two groups. These groups are unequal ; for, while the proximal centrosome always stays near the nucleus, the 
distal one makes a wide sweep at first, skirting the cell membrane before it returns to a closer course again (pl. II, fig. Io). It always happens, therefore, that the proximal centrosome retains the $\mathrm{X}$ complement and from eleven to fourteen of the bivalents, while the distal centrosome has only six to nine bivalents and never the X's (pl. I, figs. 3 and 4).

By early diakinesis, before centrosome movement is much advanced, it can be seen that each paired $\mathrm{X}$ is polarised by its centro-

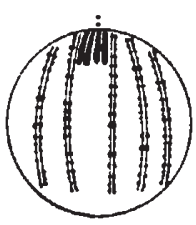

1 Paimytene

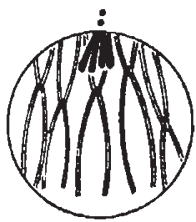

2 Diplotene

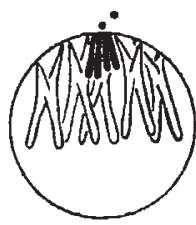

3 Diplotene

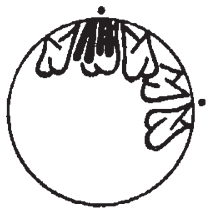

4 DiAkinesis

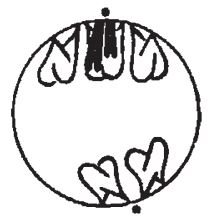

5 Diaxinesis

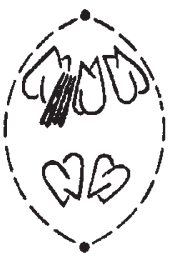

6 Polarised

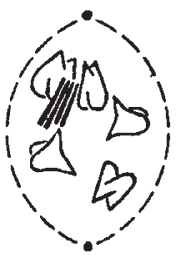

7 Transitional

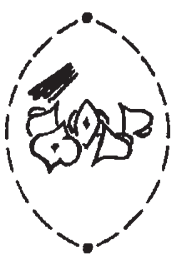

8 FulL

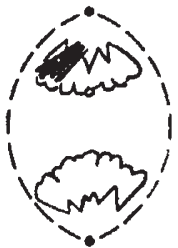

9 Anaphase

Fig.-Chromosome polarisation at the first meiotic division in Tegenaria.

mere end. This is also true of most of the bivalents of any complement, and no case was seen in which it was the distal chromosome ends which were demonstrably polarised. It is likely, therefore, that it is always the heterochromatin near the centromere that is attracted to the centrosomes.

The two groups follow their respective centrosomes round, and by late diakinesis they are on nearly opposite sides of the nucleus, held closely against the nuclear membrane (pl. II, figs. II and I2). Spindle formation follows immediately upon this stage; there is no typical diakinesis, though occasional nuclei were seen in $T$. domestica in which polarisation was relaxed sufficiently to give an almost normal dispersal of bivalents.

\section{(iii) Polarisation on the spindle}

After spindle formation chromosome polarisation is still completely maintained for a time, delaying metaphase co-orientation of the centromeres. In contrast to diakinesis the chromosomes of each group are now ranged in a semicircle round their pole so that all bivalents are the same distance from the centrosomes. This distance 
is much greater than that which separated the autosomes nearest the centrosomes from them during prophase (pl. II, $c f$. figs. II and 14). Surface repulsion between the chromosomes is reduced, and in this respect they are in a condition comparable to prometaphase. In the sectioned material cells at this stage showed so striking an imitation of anaphase that they were at first wrongly seriated ( $c f$. Wallace, Igog, fig. 26). In lacmoid squashes, however, there is no danger of confusion ( $c f$. pl. I, fig. 4, with pl. II, figs. I3 and I4). I have called it the Polarised Metaphase.

After this delay the bivalents react to the spindle. They do not, however, react together but in turn, and each bivalent reaches a metaphase condition at a different time. Thus the metaphase plate is assembled gradually, by a successive depletion of the polarised groups, each bivalent remaining at the same constant distance from its centrosome until its turn comes, when its centromeres first coorientate axially and then undergo congression by movement into the equatorial plane.

The progress of congression is clear and significant. The order in which the bivalents move down to metaphase from the poles appears to depend largely on the position of their single chiasma, and consequently on the distance separating their centromeres. The bivalents that co-orientate first are in general those with the most distal chiasmata, and these bivalents congress on or near the axis of the spindle (plate II, figs. I5 and I6). The last bivalents to react are those with an extremely proximal chiasma, in which the centromeres are very close together; these bivalents move down the spindle in an outside position and congress on the edge of the plate (plate II, figs. I7 and I8). I have called any cell with polarised and metaphase bivalents co-existent on its spindle a Transitional Metaphase ( $c f$. Wallace's fig. 23). Transitional metaphases representative of all stages in the change are seen, though the process seems to slow down as full metaphase is approached.

These pre-metaphase stages are not instantaneous conditions. They are stable and lasting. Polarised metaphase, transitional metaphase and full metaphase are seen in the proportions of about $3: 6: \mathrm{r}$, and it is quite clear that the spindle is fully formed from the first. The polarised metaphase must be a stable phase during which the chromosomes are at rest on the spindle, and the large number of transitional metaphases observed indicates that the change to full metaphase occurs only slowly.

The X's never congress equatorially on the first division spindle, and remain apparently polarised until late anaphase (pl. II, figs. I6-20). They are thus controlled throughout the division by the centrosome that was proximal at pachytene, and at telophase they are included in the same nucleus. During both polarised and transitional metaphase they are further from the centrosomes than are the autosomes 
(pl. I, figs. 4 and 5). They are occasionally separated, though always on the same side of the spindle.

At telophase a non-disjoined bivalent is sometimes seen near one pole. This is probably due to a delay in congression. No irregularities were ever observed in the behaviour of the X's.

The second divisions show no polarisation.

\section{SEX DETERMINATION}

All reports agree that the multiple $\mathrm{X}$ chromosomes in spiders are wholly heterochromatic and of exactly the same length, and that, though they remain side by side during the first meiotic division, they never form chiasmata. White, in his review of multiple sex chromosomes (194I), suggests on this evidence that the X's are completely homologous and that true pairing and crossing-over is only prevented by their being heterochromatic. In the Tegenaria species examined the two or three X's are of different lengths. Since the autosome number is constant there has evidently been an increase in number within the $\mathrm{X}$ complex, probably from an XO system, but that they have differentiated is clearly shown by these differences in length. Such differences could not be maintained with regular pairing in the homozygous sex (to which there is no known exception) if the different X's did not form separate bivalents. Nor could they form regular bivalents in the female unless they were qualitatively as well as quantitatively differentiated. The association of X's in the male, on the other hand, can be accounted for by their strong polarisation to the same centrosome, combined perhaps 'with heterochromatic stickiness; though homology may play a part, it is not necessary to account for their pairing.

No meiotic divisions were obtained in females, but the six X's in mitoses of a $T$. domestica female show that sex is determined by an $\mathrm{X}_{1} \mathrm{X}_{2} \mathrm{X}_{3}: \mathrm{X}_{1} \mathrm{X}_{1} \mathrm{X}_{2} \mathrm{X}_{2} \mathrm{X}_{3} \mathrm{X}_{3}$ mechanism in $T$. domestica and $T$. derhamii, and in $T$. atrica probably by an $\mathrm{X}_{1} \mathrm{X}_{2}: \mathrm{X}_{1} \mathrm{X}_{1} \mathrm{X}_{2} \mathrm{X}_{2}$ mechanism.

\section{CONTINUOUS POLARISATION}

\section{(I) The control of $X$ segregation}

Chromosome attraction to the centrosomes is general in animal meiosis. Descriptions have been given by Gelei (I92I), and especially by Janssens (1924) and Darlington (1936) in spermatogenesis in the Acridine, where it can be observed under very favourable conditions. Its typical features may be summarised as follows :-

(a) The autosomes are polarised by one or both ends, and the ends affected are heterochromatic.

(b) The $\mathrm{X}$ is not so strongly polarised as the autosomes and may be unpolarised. 
(c) The primary polarisation pattern may be modified by polarisation of a different nature-stickiness of heterochromatin, particularly of the X.

(d) Chromosome-centrosome polarisation lapses at the end of pachytene. Heterochromatic stickiness does not.

The concentration of chromosome ends occurs at leptotene, and it is reasonable to suppose, as Gelei (192r) has suggested, that polarisation is usually an adaptation which favours regularity of pairing. Beyond that it does no more.

Tegenaria differs in four ways: (i) the X's are more strongly polarised than the autosomes, (ii) polarisation is prolonged until anaphase, (iii) the X's are always strongly attracted to one centrosome while the other moves in such a way as to leave them together, (iv) heterochromatic stickiness of the autosome is much reduced and never modifies the primary pattern. Thus in Tegenaria it seems that polarisation is secondarily utilised to keep the X's together and to ensure their correct segregation at anaphase, and that the special features are adaptations to this secondary function.

\section{(ii) Secondary effects of the mechanism}

The action of the centrosomes on the chromosomes is apparently indiscriminate, for the autosomes as well as the X's remain polarised both in the nucleus and on the spindle. The consequences of this secondary effect and its implications will now be considered.

\section{In the nucleus}

Morse (r 909 ) has described a type of polarisation in cockroaches that is prolonged until metaphase, and Suomalainen (1946) has shown that in these species the unusual appearance of the prophase bivalents is due to the centromere and chromosome repulsion not being sufficient to open out the loops between chiasmata. White (1938) and Hughes-Schrader (1943) have described what is probably the same condition in several mantid species, and here also polarisation lasts until metaphase.

In a normal meiotic prophase centromere and chromosome repulsions are suddenly set up within bivalents at the onset of diplotene, when polarisation in most animals ceases. Now repulsions within bivalents must be correlated with those between bivalents, and it seems likely that it is the sudden increase in intra-bivalent repulsions which breaks up polarisation at this stage. If these repulsions are reduced, as in cochroaches and mantids, then polarisation continues. And in Tegenaria we must assume that, in order that the X-chromosomes may be kept together, the repulsions are so adjusted as not to be sufficient to give the dispersal characteristic of typical diplotene and diakinesis stages, and polarisation is therefore caused to continue. 
What attraction is concerned in the polarisation of chromosome ends? In Tegenaria the chromosomes follow the centrosomes when they move round the nuclear membrane. This has also been observed in other cases where polarisation lasts late into prophase-in cockroaches (Morse, I909), in mantids (White, I938, and HughesSchrader, 1943), and in Anisolabis (Schrader, 1941). Evidently, then, the attraction is to the centrosomes. Moreover, Wickbom (i 945) finds in Triturus that, following colchicine injections, there is a failure of chromosome polarisation during prophase in the primary spermatocytes, and he interprets this failure as due to an effect of the treatment on the centrosome. What is it then in the chromosome end that is attracted to the centrosome? In Tegenaria as in other animals the polarised ends are heterochromatic at certain stages. Ribbands (I94I) has suggested very plausibly that the proximity to the nuclear membrane of chromosome regions heavily charged with nucleic acid is due to the rapid synthesis of nucleotides by these regions. During polarisation the centrosome, which must itself be a nucleoprotein, is undergoing rapid growth and is organising the spindle. Chromosome polarisation is therefore perhaps brought about by the chemical co-operation of the bodies which appear to attract one another.

\section{On the spindle}

The rigidity of the polarising mechanism also introduces some unusual features into the behaviour of the autosomes on the spindle. Normally, when the meiotic spindle forms, the centromeres of each chromosome association show two characteristic reactions to the new medium - they co-orientate, and they undergo congression in the equatorial plane (Darlington, 1937). These reactions are more or less coincident with spindle formation. The behaviour of the bivalents in Tegenaria at this stage, as we have seen, is remarkable in two respects. First, their reaction to the spindle is delayed and they remain for a time attracted to the centrosomes. Secondly, when they do react, they do not do so simultaneously but instead successively, and the full metaphase plate is assembled by degrees from the two polarised groups.

A situation in which chromosomes are, or appear to be, attracted to the poles of the spindle merits examination. During prophase the polarising attraction has been strong enough to draw the bivalent ends against the nuclear membrane, close to the centrosomes. With the invasion of the nucleus by the spindle at the end of diakinesis, we find that, though polarisation continues, the bivalents take up positions on the spindle in which they are further from the centrosomes than before (pl. II, $f f$. figs. I I and I4); evidently when the bivalent ends are in the orientated spindle medium they cannot approach the centrosomes so nearly as when they are outside it. Clearly the poles are repelling the centromeres within the meiotic spindle in Tegenaria 
from the first, but during the polarised stage this repulsion must be in equilibrium with the attractive force between chromosome ends and centrosomes.

\section{THE TACTOID HYPOTHESIS}

What then is the nature of this equilibrium? Bernal (1940) has advanced a hypothesis to account for spindle formation and for the consequent chromosome reactions, based upon the assumption that the spindle is a natural tactoid. Tactoids are the characteristic spindle-shaped gels that appear under suitable conditions in sols such as those of tobacco mosaic virus. The shape of the tactoid is due to the modification of the parallel orientation of the axially arranged molecules on either side of the equatorial plane so that they converge upon the poles. It is only with long needle-shaped molecules that the necessary surface tension conditions exist at the poles which bring this modification about. Enclosures of unorientated medium in the tactoid take on a tactoid shape and are called negative tactoids. They tend to move into the equatorial plane and into the axis of the positive tactoid because they cause least distortion of the molecular orientation in this position.

Bernal suggests that the centrosome first induces an orientation of the long molecules radiating from itself to give an aster. It then divides as a result of the instability of the system, and a tactoid is formed, which increases in size by the growth of its molecules and envelopes the nucleus as the centrosomes move round. The centromeres (at least when active) are assumed to be able to cause local dissolution of the long molecules, and when they come into the spindle medium they produce small negative tactoids which move into the equatorial plane to give metaphase congression and orientation. Anaphase movement is brought about by the subsequent growth of each negative tactoid, and the movement apart of the two daughter centromeres within it.

The birefringence shown by the living spindle (Schmidt, 1937) and its general tendency to contract transversely in hypertonic solutions (Belar, 1929) support the view that it is a tactoid. There is little direct evidence for the existence of the postulated negative tactoids. Now, however, indirect evidence is available. There are the observed effects of experimental variables such as cold and colchicine (Barber and Callan, 1943), and observations on naturally aberrant mechanisms such as those of the autosomes and sex chromosomes on the spindle in Tegenaria.

On Bernal's theory each bivalent should begin to react immediately the nucleus was penetrated by the orientated medium, owing to the polar repulsion of the negative tactoid formed by the centromere pair. But in Tegenaria the attraction between chromosome ends and centrosomes still seems to operate, since the chromosomes take up positions 
on the spindle which are the points of equilibrium between these two forces. On the assumption that the repulsion of negative tactoids by the poles increases steeply towards the poles, the chromosomes might well be stabilised in the two semicircular groups around these poles.

At transitional metaphase this equilibrium is disturbed by the individual reaction of each bivalent to the spindle. The order in which the bivalents move on to the plate is significant. Bivalents with terminal chiasmata tend to congress first. This means that movement is influenced by the distances between the pairs of centromeres-that is, presumably, by the size of the negative tactoid formed by each bivalent.

The relative uniformity of autosome size in Tegenaria is probably an adaptation to reduce these differences as much as possible.

The behaviour of the $\mathrm{X}$ group may be compared with that of the type of univalent which never reaches the plate at all, and is included undivided in one of the telophase nuclei in the same way as the Xchromosome in the Acridina. It does not move on to the plate because, having a single undivided centromere, it has no negative tactoid.

This interpretation is not offered as the only physical explanation of the facts. But it is clear that, from the conception we have of the cell spindle as a liquid crystal of tactoid type, it is possible to explain aberrant as well as normal types of cell division.

\section{SUMMARY}

I. Three species of Tegenaria have 20 pairs of autosomes. The sex chromosome number varies according to the species-2 or $3 \mathrm{X}$ 's in the males and twice the number in the females.

2. At meiosis in the males the $X$ 's are strongly heterochromatic; they do not form chiasmata but remain side by side, and at first telophase they are included in the same nucleus. The regular segregation of the X's is ensured by their being held near the same centrosome throughout the first division.

3. The autosomes are attracted to one centrosome or the other during prophase. Consequently there is no typical diplotene or diakinesis, and a stage resembling anaphase precedes metaphase. The autosomes later congress on the spindle, while the X's do not. This continuous polarisation is considered to be due to a reduction or delay in the centromere and chromosome repulsions which normally disrupt polarisation at diplotene.

4. The behaviour of the autosomes and X's on the first meiotic spindle is shown to be describable in terms of the tactoid theory of spindle structure and the known cycles of centromere activity.

I wish to thank Dr P. T. Thomas for advice during the preparation of this paper. 
Prate I.-Tegenaria atrica (except fig. 6, T. domestica); first division in the Sperm mother cell. Acetic-alcohol, Lacmoid. $\times 1400$.

Fic. I.-Pachytene.

Fic. 3.-Diakinesis: $2 \mathrm{X}$ 's in top group.
FIG. 2,-Diplotene.

FIG. 4.-Polarised metaphase : 2 X's in top group.

Lic. 5.-Transitional metaphase. Fig. 6.-Full metaphase with 3 X's.

Fic. 7.-Anaphase.
Fic. 8.-Interphase: $2 \mathrm{X}^{\prime} \mathrm{s}$ in top nucleus. 
- 150

4. 5 , 4

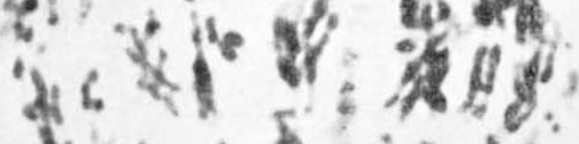

$x+5$ ?

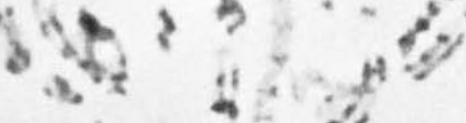

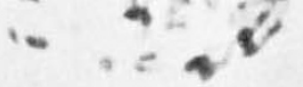

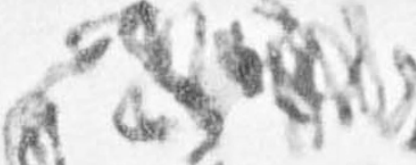

(4) 1

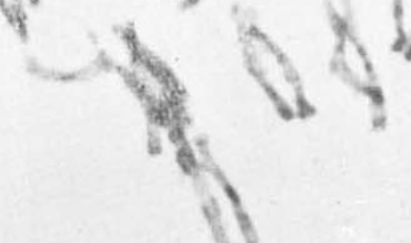

- a $y^{k}$

-990\%

v

Jor.

$\mu$

ริ

lis't

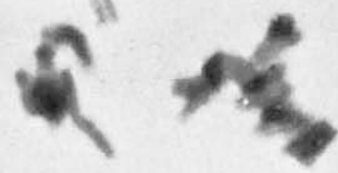

TnMa

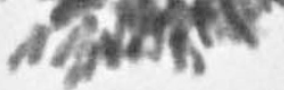

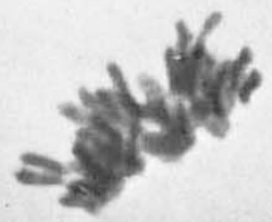

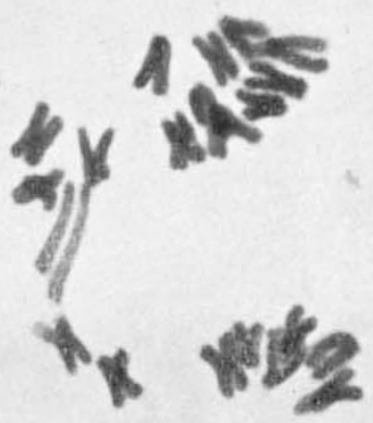

nI

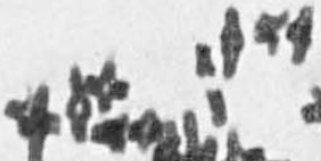
को

hes.

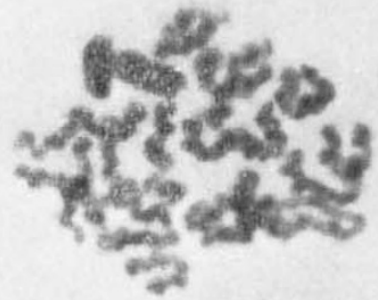

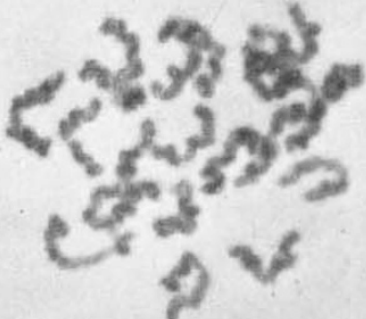


Plate. II.-The same : ${ }_{2} B D$, Crystal Violet. $\times 2600$.

FIG. 9.-Pachytene: proximal FIG. Io.-Diplotene: movement FIG. II. - Diakinesis: and distal centrosomes.

of the distal centrosome.

two polarised groups of chromosomes.

FIG. I2. - Diakinesis: two Fics. 13 and 14 .--Polarised metaphase. polarised groups of chromosomes.

Figs. 15-17.-Transitional metaphase: stages in the formation of the metaphase.

FIG. I8.-Transitional meta- FIG. 19.-Full metaphase. FIG. 20.-Anaphase. phase: the last stage. 


\section{REFERENCES}

BARBER, H. N., and GALlAN, H. G. I943. MORSE, M. 1909.

Proc. roy. SOC. $131,258$.

Arch. Zellforsch. 3, 483 .

BELAR, K. I929.

Arch. Entw. Org. 118, 359.

PAINTER, T. S. 1914.

Zool. Fahrb. 38, 509 .

BERNAL, J. D. I940.

The Cell and Protoplasm.

Am. Ass. Adv. Sci. Symposium.

RIBBANDS, C. R. 194 I.

7. Genet. $4 \mathrm{I}, 4 \mathrm{I}$ I.

DARLINGTON, C. D. 1936.

7. Genet. 33,465 .

SCHMIDT, w. J. 1937.

Die Doppelbrechung von Karyoplasma, Zytoplasma und Metaplasma. Berlin.

DARLINGTON, C. D. 1937 .

Recent Advances in Cytology. London.

DARLINGTON, C. D., and LA COUR, L. F.

1942.

SCHRADER, F. I94I.

7. Morph. 68, 123.

SUOMALAINEN, E. 1946.

Ann. Acad. Sc. Fenn. A. IV. Io, I.

GELEI, J. 1921 .

Arch. Zellforsch. 16, 88.

WALLACE, L. B. 1909.

Biol. Bull. 17, 120.

WHITE, M. J. D. 1938.

HARD, W. L. 1939.

Proc. roy. Soc. 125, 516.

HUGHES-SCHRADER, S. 1943 .

WHITE, M. J. D. 1940.

Biol. Bull. 85, 265 .

7. Genet. $4^{2}, 143$.

JANSSENS, F. A. 1924 .

พIсквом, т. 1945 .

La Cellule 34, 135.

Hereditas 3I, $24 \mathrm{I}$. 